\title{
Quenching of internal rotations versus collisional cooling at ultralow energies for weakly interacting partners: $\mathrm{Cs}_{2}\left({ }^{3} \Sigma_{u}^{+}\right)$with ${ }^{3,4} \mathrm{He}$
}

\author{
D. Caruso,${ }^{1}$ M. Tacconi,${ }^{1}$ E. Yurtsever, ${ }^{2}$ and F. A. Gianturco ${ }^{1, *}$ \\ ${ }^{1}$ Department of Chemistry and CNISM, University of Rome La Sapienza, Piazzale A. Moro 5, I-00185 Rome, Italy \\ ${ }^{2}$ Department of Chemistry, Koç University, Rumelifeneriyolu, TR-34450 Sariyer, Istanbul, Turkey
}

(Received 9 February 2010; published 26 April 2010)

\begin{abstract}
Quantum-scattering calculations at ultralow (close to $10^{-6} \mathrm{~cm}^{-1}$ ) collision energies are carried out for the Cs dimer in its spin-stretched triplet state, interacting with helium. An ab initio potential energy surface is computed and employed, while the target molecule is kept in its ground vibrational state and several excitated initial rotational states are considered in the quantum dynamics. The highly anisotropic interaction is seen to cause, in spite of its weakness, internal energy quenching rates comparable with the efficiency of the collisional cooling of relative kinetic energies. The rates of spin-flip processes are also analyzed and compared with pure rotational quenching events.
\end{abstract}

DOI: 10.1103/PhysRevA.81.042710

PACS number(s): 34.50.-s, 34.20.Gj, 37.10.Mn

\section{INTRODUCTION}

Over the past few decades there has been quite impressive growth, both in theoretical work and in experiments, of the study of atomic and molecular processes at low and ultralow temperatures [1-3], a field of research which has remarkably increased our knowledge on a very broad variety of topics, from atomic physics [4] to a better understanding of molecular properties [5] to chemical processes [6] and even astrophysics [7]. In addition to enhancing the potentials of the spectroscopic analysis and of increasing our abilities of controlling process evolutions, cold trapped molecules have begun to play an ever-increasing role in guiding our understanding of chemical reactions, of the role of fine and hyperfine-structure interactions in such processes, and on the fundamental function of molecular symmetry in allowing condensation effects at ultralow temperatures [8-10]. However, the inability to directly apply to molecular aggregates the cooling procedures employed so successfully for atoms has initially slowed progress in this area, where the development of viable alternatives has again spurred more investigations into cold and ultracold molecular environments. In particular, the technique of buffer gas cooling has revealed itself as being a very general and fairly successful method: it was developed by J. Doyle and coworkers [11] whereby the buffer gas (helium) is itself cooled first by a criogenic device, such as a thermostat or a dilution refrigerator. Hence, the buffer gas mediates thermal contact between the partner molecule and the cryogenic device and the equilibration process that is driven by this contact results from elastic and inelastic collisions between the helium atoms and the cooling molecules: typical conditions require about 100 collisions for such thermalizations to occur [12]. Naturally, rapid thermalization is an important prerequisite of such processes, and therefore the buffer gas must have a sufficiently high density to ensure that molecules are cooled along a path shorter than the dimensions of the cell (of a few centimeters). This also means that a certain minimum temperature exists below which the number density

\footnotetext{
*Corresponding author: fa.gianturco@ caspur.it
}

of the helium buffer would be low enough to enable the thermalization process: for $\mathrm{He}$ this corresponds to about $240 \mathrm{mK}$ [11]. It follows from the above outline that a realistic knowledge of the cross sections at the operating temperatures of the cooling device is one of the important ingredients for guiding both the preparation of experimental tests and the assessment of the feasibility of the method for a particular molecular gas. More specifically, one would need to know as accurately as possible the relative sizes of the elastic collisions that drive the translational cooling of the partner molecules with respect to the superelastic collisions that quench the internal temperatures of the same molecular partner while, however, also evaporating buffer gas atoms and some of the "hot" molecules from the traps. In the present study, therefore, we have decided to examine a molecular dimer species obtained from cesium atoms, the $\mathrm{Cs}_{2}$ molecule, which is taken to have been formed in its "spin-stretched," state ${ }^{3} \Sigma_{u}^{+}$, where the atom-atom interaction is markedly weaker than that of its singlet ground state, as we shall further discuss in the next section. The above system is in turn considered to be in its ground vibrational level (but still in some excited rotational state) and to interact with ${ }^{4,3} \mathrm{He}$ atoms via an $a b$ initio constructed potential energy surface (PES) so that it becomes computationally possible to investigate those elastic and superelastic collisional processes at ultralow temperatures which are deemed to be important for an estimate of the system's efficiency in carrying out the competition between elastic and quenching processes from the thermal region to below $10^{-6} \mathrm{~K}$. The reasons why we think of it is of interest to investigate such situations are the following:

(a) The formation of cesium dimers in cold traps has been analyzed in several earlier articles [13-15] and still presents specific features which need to be further clarified: in other words, it remains a system of high current interest.

(b) The interaction of its triplet state with helium atoms, both bosons and fermions, has been rarely obtained from accurate calculations and never for a vibrating molecule in its ground state. It therefore becomes important to assess the strength and anisotropy of such interaction starting from reliable $a b$ initio methods. We therefore employ here an $a b$ initio computed PES, obtained within our group. 
(c) Nothing is known about the size of their relative cross sections at low and ultralow temperatures nor on the relative importance of elastic events versus rotational quenching processes at such low and ultralow $T$ values. We therefore carry out essentially exact quantum dynamics for this system in order to produce realistic estimates of those very quantities.

(d) The corresponding effects from the spin-rotation coupling are also unknown and the hierarchy of inelastic process sizes involving these quenching channels is not known; such features are therefore analyzed here and some specific conclusions can be drawn from the present study.

The article is organized as follows: the next section describes the calculations of the relevant PES and reports its most significant features; it also outlines the quantum dynamical equations. Section III describes our results and analyzes the relative sizes and energy dependence of the relevant cross sections for both the elastic and the quenching channels as the energy approaches the ultracold conditions mentioned before $\left(10^{-4}-10^{-7} \mathrm{~cm}^{-1}\right)$. Our conclusions are finally given in Sec. IV.

\section{INTERACTION AND DYNAMICS}

As mentioned in the previous section, the full potential energy surface describing the interaction between a vibrating cesium dimer in its ${ }^{3} \Sigma_{u}^{+}$electronic state and an He atom in its ground electronic state $\left({ }^{1} S\right)$ has been computed by us from first principles using a pseudopotential description of the Cs atoms and yielding the full interaction at the post-Hartree-Fock level of accuracy as described below.

\section{A. Ab initio electronic structure of the PES}

All $a b$ initio calculations are performed with the GAUSSIAN03 program [16], using the spin-unrestricted single and double excitations coupled-cluster method with perturbative triples, correlating only the valence electrons. We use Jacobi coordinates $(R, r, \theta)$ to describe the potential surface of the $\mathrm{HeCs}_{2}$ complex, where $R$ is the intermolecular distance of the He atom from the center of mass of $\mathrm{Cs}_{2}, r$ is the bond length of $\mathrm{Cs}_{2}$, and $\theta$ is the angle between the $\mathbf{R}$ and $\mathbf{r}$ vectors. Angles are incremented by $10^{\circ}$ from $0^{\circ}$ to $90^{\circ}$ and distances extended out to $24 \AA$ using different spacings between $R$ values depending on its orientation. The range of the $\mathrm{Cs}_{2}$ bond length spanned 10 different values from $5.8 \AA$ to $10 \AA$, including the $r_{e q}$ value $(6.235 \AA)$. In order to asses the quality of the calculations, different choices of basis set expansions were implemented and tested before carrying out the final evaluation. For instance, the potential curve of the $\mathrm{Cs}_{2}$ molecular partner was generated by selecting five effective core potentials (ECP) from different groups within the $\operatorname{CCSD}(\mathrm{T})$ approach: the LANL2DZ [16] which further uses 52 primitive Gaussian-type orbitals (GTOs) contracted to 24 for the valence electrons; the Hay-Wadt [17] and the ECP46MWB [18] that use for the valence electrons 46 and 50 primitive GTOs contracted to 16 and 34, respectively; the ECP46MDF [19] that uses now 270 primitive functions contracted to 192 and the CRENBL [20] effective potential that uses 88 primitive GTOs contracted to 80 . In Table I we present the values of $r_{e q}$ and of the well depth, $D_{e}$, obtained using a sampling of the above-mentioned ECPs, together
TABLE I. Values of $r_{e q}$ and of the well-depth, $D_{e}$, computed here and compared with experiments and with earlier calculations.

\begin{tabular}{lcc}
\hline \hline & $r_{e q}(\AA)$ & $D_{e}\left(\mathrm{~cm}^{-1}\right)$ \\
\hline & Present results & \\
ECP46MWB & 7.8 & 91.38 \\
ECP46MDF & 6.5 & 229.61 \\
CRENBL & 6.82 & 211.68 \\
Expt. Xie et al. [21] & 6.235 & 279.349 \\
& Earlier calculations & \\
Foucrault et al. [22] & 6.355 & 233 \\
Li et al. [23] & 6.30 & 295 \\
\hline \hline
\end{tabular}

with previous estimates of those magnitudes. The most recent potential energy curve, derived from fluorescence measurements [21], presents values of $6.235 \AA$ and $279.349 \mathrm{~cm}^{-1}$, in accordance with some earlier theoretical estimates [22,23]. In our calculations the CRENBL turns out to produce a realistic description of the dimer triplet potential minimum features (6.8 $\AA$, and $\left.211.683 \mathrm{~cm}^{-1}\right)$, while keeping the computational effort within acceptable limits. In fact, the latter values are in line with earlier estimates on this system, as $6.35 \AA$ and $233 \mathrm{~cm}^{-1}$ [22]. The CRENBL choice already includes $5 s$ and $5 p$ electrons in the definition of the valence space allowing an explicit treatment of core-valence electron correlation in subsequent calculations. Keeping this requirement in mind, the particular ECP choice to describe the dimer is expected to be realistic for the overall $\mathrm{He}-\mathrm{Cs}_{2}$ interaction, although slightly deeper wells would more accurately reproduce experiments [21]. The basis set for the He atom was also varied from cc-pVQZ to cc-pV5Z to aug-cc-pV5Z and then to d-aug-ccpVQZ and d-aug-cc-pV5Z. In this way, the interaction energies using the supermolecular approach, $\Delta E=E_{\mathrm{HeCs}_{2}}-E_{\mathrm{Cs}_{2}}$, were obtained. The best choice, including the well known counterpoise correction [24], turned out to be the d-aug-ccpVQZ in terms of strength of the very weak interaction and feasibility of computational times, producing variations of the minimum values of the attractive wells which are essentially coincident with those given by the d-aug-cc-pV5Z choice. The use of the d-aug-cc-pV5 basis set slightly improves the quality, obtaining lower interaction energies with respect to d-aug-cc-pVQZ: the results, however, are too costly for our large number of computational points (see before). We have found no data on the full rotovibrational potential for this system: it is therefore difficult to make an assessment on the quality of our results. The comparison between the bound vibrational levels yielded by our curve and those provided by the recent estimates [25] indicates differences around the bottom of the well region, with us producing a zero-point energy for the $v=0$ level of $4.75 \mathrm{~cm}^{-1}$, to be contrasted with a value of $5.7 \mathrm{~cm}^{-1}$ from the potential of Ref. [25]. Since we are interested at the moment on the comparative behavior of rotation-quenching collisions with respect to those for the collisional cooling cross sections, we decided to average the computed PES over the ground vibrational level of the target as an initial computational choice

$$
V_{00}(R, \theta)=\int \varphi_{0}^{*}(r) V(R, r, \theta) \varphi_{0}(r) d r
$$




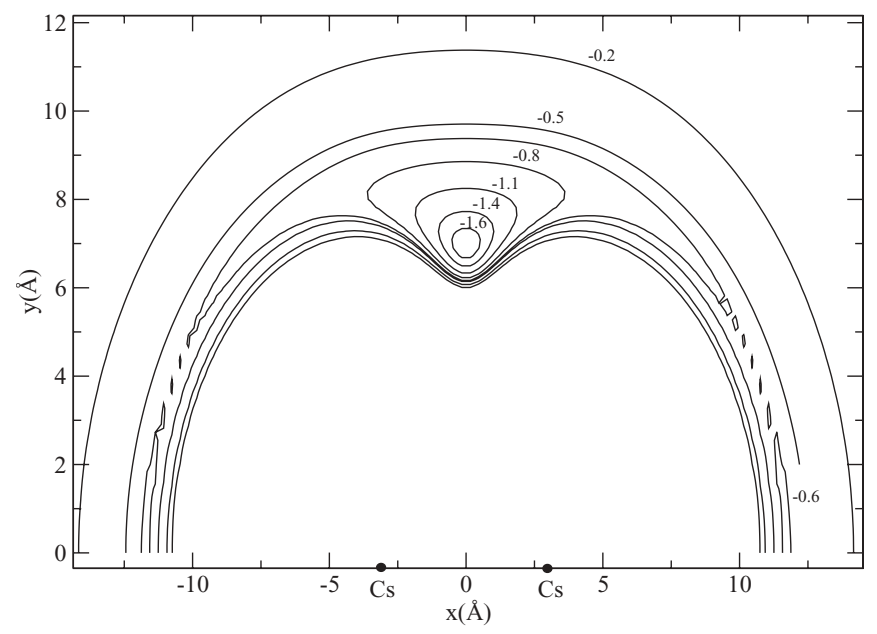

FIG. 1. Computed PES isolines as a function of the distances from the $\mathrm{CS}_{2}$ center of mass on the $x y$ plane. The $\mathrm{V}_{00}$ potential is given in $\mathrm{cm}^{-1}$ and the distances are in $\AA$. The well depth indicated in the figure is $1.6 \mathrm{~cm}^{-1}$ (see text for details).

so we treat the $\mathrm{Cs}_{2}$ target not as a rigid rotor but as a vibrating target averaged over its vibrational ground state. This is a reasonable approximation given the type of experimental conditions we wish to explore with calculations, since we expect that the molecules in the trap could be vibrationally cold as we analyze their rotational quenching during the interface with the buffer gas. That the overall interaction is indeed very weak could be gleaned from the pictorial representation of the PES given by Fig. 1, where the energy isolines are reported around the $\mathrm{Cs}_{2}$ molecular target.

One clearly sees from that figure how the largest well depth is located at the T-shaped geometry and reaches a value of about $1.6 \mathrm{~cm}^{-1}$, while the collinear orientation yields a well depth of only about $0.6 \mathrm{~cm}^{-1}$. Thus, the system is indeed very weakly interacting and supports only one bound state for ${ }^{4} \mathrm{He}$ in the $J=0$, nonrotating state of the complex [26]. On the other hand, it is also highly anisotropic since it shows very marked changes of strength and shape as the orientational angle is changed. This is more immediately grasped by looking at the coefficients of the corresponding multipolar expansion

$$
V_{00}(R, \theta)=\sum_{\lambda} V_{\lambda}^{00}(R) P_{\lambda}(\cos \theta),
$$

where the familiar coefficients provide a measure of the strength and range of the anisotropy of the full interaction. As is well known, the coefficients are obtained by matrix inversion using the inverse matrix of Legendre polynomials $\mathbf{P}^{-1}$, with $P_{k \lambda}=P_{\lambda}\left(\cos \theta_{k}\right)$. This procedure constitutes the collocation procedure over the grid of $\theta_{k}$ angular values after the integration of $r$ given by Eq. (1). Figure 2 reports the first five coefficients (only even labels allowed) and provides clear indication on their relative values and on the smoothness of the present procedure for the vibrationally averaged PES. The vibrational label of each multipolar coefficient is not given for reasons of clarity.

The shapes of the reported curves in Fig. 2 show immediately that the $V_{2}$ coefficient is nearly as strong as the spherical term, $V_{0}$, thereby indicating the marked anisotropy of this

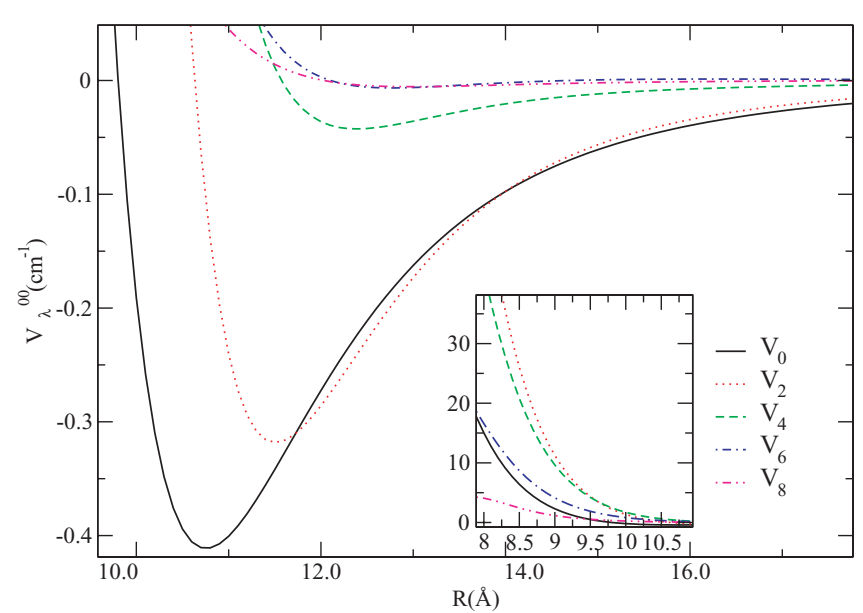

FIG. 2. (Color online) Computed multipolar coefficients from the expansion of Eq. (2). See main text for details. The vibrational index is omitted in the panel for reasons of clarity.

interaction. The $V_{4}$ coefficient also shows the presence of a well, although the higher $\lambda$ are already mainly repulsive, with only an insignificant attractive tail of their interaction. On the whole, therefore, substantial spatial extension of at least two anisotropic coefficients will condition the dynamical outcome of the rates following the internal rotation quenching processes that we will examine below; the calculations from the figure indicate that we should expect marked effects on the final distribution into the various rotational states of this molecular partner because of the remarkable strength of the anisotropic features reported by our calculations and in Fig. 2. It is also worth noting here that the shapes of the multipolar curves shown by the inset in Fig. 2 indicate that, at ultralow collision energy the $V_{2}, V_{4}$, and $V_{6}$ coefficients have more external turning points with respect to the spherical potential. In terms of collisional quenching efficiency one therefore expects substantial inelastic coupling in the system. This is in keeping with what we found earlier for the $\mathrm{Li}_{2}$ spin-stretched case in collision with He [27].

\section{B. Spin-rotation coupling scheme}

In the case of the triplet state of the target molecule, we have three levels available for each total angular momentum: the rotational levels are in fact split by spin-rotation coupling. In the pure Hund's case (b) the electronic spin momentum $S$ couples with the nuclear rotational angular momentum $N$ ( $N=R$ for a $\sigma$ state) to form the total angular momentum $j$, given by

$$
j=N+S \text {. }
$$

Hence, in this coupling scheme, each $j$ level (with $j \geqslant 1$ ) is split into three sublevels, $F_{1}, F_{2}$, and $F_{3}$, and the corresponding rotational wave function can be written as

$$
\begin{gathered}
\left.\left|F_{1} j m\right\rangle=N=j-1, \operatorname{Sj} m\right\rangle \\
\left.\left|F_{2} j m\right\rangle=N=j, \operatorname{Sj} m\right\rangle \\
\left.\left|F_{3} j m\right\rangle=N=j+1, \operatorname{Sj} m\right\rangle,
\end{gathered}
$$

where $m$ is the projection of $j$ along the space-fixed $z$ axis. The rotational levels of the molecule may be labeled not only by the above quantum numbers defined in the Hund's case (b) 
TABLE II. Computed energies of the rotational levels of the $\mathrm{Cs}_{2}\left({ }^{3} \Sigma_{u}^{+}\right)$near the bottom of the energy scale. See main text for details.

\begin{tabular}{lllc}
\hline \hline$N$ & $j$ & $F$ & Energy $\left(\mathrm{cm}^{-1}\right)$ \\
\hline 0 & 1 & $F_{1}$ & 0.0 \\
2 & 1 & $F_{3}$ & 0.0310 \\
2 & 3 & $F_{1}$ & 0.0321 \\
2 & 2 & $F_{2}$ & 0.0350 \\
4 & 3 & $F_{3}$ & 0.1075 \\
4 & 5 & $F_{1}$ & 0.1080 \\
4 & 4 & $F_{2}$ & 0.1110 \\
6 & 5 & $F_{3}$ & 0.2270 \\
6 & 7 & $F_{1}$ & 0.2274 \\
6 & 6 & $F_{2}$ & 0.2305 \\
\hline \hline
\end{tabular}

but also by their parity index $\varepsilon$. The levels in molecules of odd multiplicity with parity index equal to 1 are labeled $\mathbf{e}$, and those with parity index equal to -1 are labeled $\mathbf{f}[28,29]$. However, we shall omit this index in the following discussion since we shall not be using it in our analysis. For a given $N$ value, the energies of the three levels in Eq. (4) are given by [29]

$$
\begin{gathered}
E_{j=N+1}=B N(N+1)-D N^{2}(N+1)^{2}-\frac{2 \lambda N}{3(2 N+3)}+\gamma N \\
E_{j=N}=B N(N+1)-D N^{2}(N+1)^{2}+\frac{2 \lambda}{3}-\gamma \\
E_{j=N-1}=B N(N+1)-D N^{2}(N+1)^{2} \\
-\frac{2 \lambda(N+1)}{3(2 N-1)}-\gamma(N+1),
\end{gathered}
$$

where $B\left(=0.0054 \mathrm{~cm}^{-1}\right)$ [30] is the rotational constant, $D$ is the centrifugal distortion, $\lambda$ is the spin-spin interaction, and $\gamma$ is the spin-rotation interaction. Note the smallness of the $B$ constant, hence the very close energy spacings in Table II. Hence we did not include the centrifugal distortions since we expect $D$ to be an even smaller contribution to the energy spacings generated by $B$ only.

\section{Cross sections from quantum scattering calculations}

We shall not repeat here the well-known formulation of the coupled-channels approach to quantum inelastic scattering $[31,32]$ but shall provide below only a short outline of it. The familiar set of coupled, second-order homogenous differential equations reads

$$
\left\{\frac{d^{2}}{d R^{2}}+\mathbf{k}^{2}-\mathbf{V}-\frac{\mathbf{l}^{2}}{R^{2}}\right\} \Psi=0,
$$

where $\left[\mathbf{k}^{2}\right]_{i j}=\delta_{i j} 2 \mu\left(E-\varepsilon_{i}\right)$ is the diagonal matrix of the asymptotic (squared) wave vectors, $\left[\mathbf{l}^{2}\right]_{i j}=\delta_{i j} l_{i}\left(l_{i}+1\right)$ is the matrix representation of the square of the orbital angularmomentum operator, and $\mathbf{V}=2 \mu \mathbf{U}$ is the potential coupling matrix. $\Psi$ is the solution matrix which contains the radial channel components of the scattering wave function. In the asymptotic region this solution matrix can be written in the form

$$
\Psi(R)=\mathbf{J}(R)-\mathbf{N}(R) \mathbf{K},
$$

where $\mathbf{J}(R)$ and $\mathbf{N}(R)$ are matrices of Riccati-Bessel and Riccati-Neumann functions. Using them we can now extract the scattering matrix $\mathbf{S}$ from propagating Eq. (6) to the asymptotic region of interaction and, therefore, the relevant cross sections: for all present calculations we have employed the code developed in our group [32] where, at short range, the propagator was given by a log-derivative algorithm while at long range it employed the modified variable-phase propagator. In the integration of the coupled equations we have used a varying number of the total angular-momentum $J$ values depending on the collision energy: for the largest energies, at most seven total angular-momentum values were needed for convergence and we have included all rotational states up to $j_{\max }=20$ for the present system. We have performed calculations for collision energies from $10^{-8}$ to $1.0 \mathrm{~cm}^{-1}$, and for all initial rotational states up to $j=6$. The triplet case included all terms coming from spin-spin $(\lambda)$ and spin-rotation $(\gamma)$ coupling contributions within the well-known equations already presented before. As shown by Eq. (5), the $\gamma$ and $\lambda$ constants are responsible of the magnitude of the fine-structure splitting of the $\mathrm{Cs}_{2}$ rotational levels. To the best of our knowledge, however, there have not been to date experimental or computational attempts at evaluating these quantities for the ${ }^{3} \Sigma_{u}^{+}$electronic state of the $\mathrm{Cs}_{2}$ molecule. We have overcome this somewhat surprising lack of information about the above electronic state of the cesium dimer by using the computational methodology described in Ref. [33] and implemented in the 2.0 release of the DALTON program package [34]. More precisely, the method described by Ref. [33] allows the ab initio evaluation of the electronic $g$ tensor which is related to the $\gamma$ spin-rotational constant by the Curl relations: $\gamma=-B \times \Delta g$. Furthermore, the $\lambda$ constant can be directly calculated within the same theoretical and computational framework [33]. We have therefore generated MCSCF and ROHF wave functions in order to evaluate the $g$ tensor (MCSCF) and the $\lambda$ constant (ROHF) of the $\mathrm{Cs}_{2}{ }^{3} \Sigma_{u}^{+}$. In order to make more feasible the calculation of the $\mathrm{Cs}_{2}$ wave functions, an effective core potential (ECP) has had to be used. We have chosen the quasirelativistic ECP ECP46MWB from the Stuttgart group [18-20] along with the primitive Gaussian basis set $(6 s 6 p 1 d)$ contracted at $[4 s 4 p 1 d]$. Both the Gaussian basis set and the ECP46MWB are part of the built-in basis set library distributed with the DALTON program. During the calculation, the internuclear distance of the $\mathrm{Cs}_{2}$ has been taken to be the experimental equilibrium distance of $6.235 \AA$. Within the computational method outlined above we finally found that $\gamma=1.6362 \times 10^{-7} \mathrm{~cm}^{-1}$ and $\lambda=3.2455 \times 10^{-3} \mathrm{~cm}^{-1}$, i.e., both coupling constants turn out to be very small.

We have also calculated the global cooling rates as a function of the initial $j$ state of the molecular partner [27,35]

$$
\Gamma_{j}(T \rightarrow 0)=\frac{4 \pi \beta_{j} \hbar}{\mu},
$$

where $\beta_{j}$ is the imaginary part of the scattering length which can be obtained from the total quenching cross section, $\sigma_{j}^{i n}$, in the limit of vanishing kinetic energy [36]

$$
\beta_{j}=\lim _{k_{j} \rightarrow 0} \frac{k_{j} \sigma_{j}^{i n}}{4 \pi} .
$$


The present rate coefficients correspond to the limiting situation of zero temperature associated with each of the scattering processes. We shall further discuss below the behavior of the above rates at ultralow energies, vis-à-vis the corresponding behavior of the relevant individual cross sections analyzed in the following section.

\section{Dynamical results and discussion}

We have carried out a computational analysis of the elastic and quenching collisions down to $10^{-8} \mathrm{~cm}^{-1}$ of relative translational energy for both helium isotopes, with the initial states of the molecular rotor associated with $N=2,4$, and 6 . These values should provide a sufficient range of data to give us indications on the general behavior of the processes at such ultracold conditions. In the case of scattering with ${ }^{3} \mathrm{He}$, we have not included the additional coupling between the nuclear spin of the fermionic partner and that of the electronic structure of the dimer it was considered to be too small to be included in the present treatment.

The results reported in the three panels of Fig. 3 describe the different cross sections obtained with ${ }^{4} \mathrm{He}$ as partner and labeled specifically in the figure. The initial state considered is, as an example, the excited target state with $N=2$, and the three components due to the fine-structure splitting are also shown; the three panels refer, in fact, to the three possible initial values of $j(1,2$, and 3$)$ associated with each of the reported states. The following comments could be made from our findings:

1. Due to the rather weak interaction between $\mathrm{Cs}_{2}\left({ }^{3} \Sigma_{u}^{+}\right)$ and the helium atom (see previous section) the corresponding elastic cross sections are seen to reach the Wigner's regime [37], i.e., to be independent of the collisional energy on the $\log -\log$ scale, at energies around $10^{-2} \mathrm{~cm}^{-1}$, and for all cases examined.

2. The total quenching cross sections (i.e., those combining rotational quenching and spin-flipping between fine-structure levels) are all fairly large, due to the strong angular anisotropy of the intermolecular forces, as described earlier and as shown by Fig. 2 .

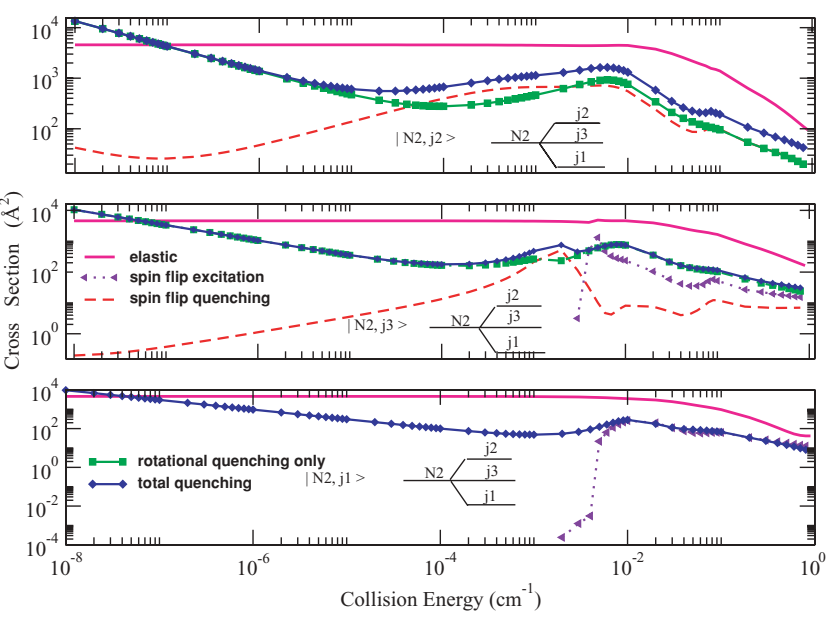

FIG. 3. (Color online) Computed inelastic cross sections starting from the level with $N=2$. See main text for details.
3. Because of the limited number of available internal states for the quenching process the superelastic cross sections that originate from $N=2$ are not very large at these low energies, hence they become larger than the translational cooling probabilities (elastic cross sections), only when the relative energy goes down to the ultralow value of $10^{-7} \mathrm{~cm}^{-1}$; furthermore, pure rotational quenching events largely dominate the inelastic processes.

4. The separate contributions of the "spin quenching" cross sections, i.e., those cross sections which are associated with spin-flipping processes within the fine-structure manifolds shown in the panels, are seen to drastically reduce in size as the collision energy is reduced and are expected to resume a Wigner-type divergent behavior on the familiar log-log scale only at much lower energies. This feature, common to all our present data, is related to the presence of additional barriers in the outgoing channels which are coupled to the initial, all-important, $l=0$ channel. In most cases, in fact, the downward transitions to different $j$ values are associated with contributions in the outgoing channels from relative angular momenta that are larger than zero [38]. This effect causes the outgoing wave function to die below the barrier height and therefore to prevent final countributions to the outgoing flux to go to that particular state. Hence the strong reduction of the corresponding pure spin-flip quenching processes which we see in the panels of Fig. 3. The panels shown in Fig. 4 further provide an additional, pictorial view of such blocking barriers for a few indicative states of the molecular target.

5. The excitation of fine-structure levels, because of the very small values of the energy involved, are indeed possible at low collision energies but become rapidly closed as the relative translational energy decreases. The lower two panels of Fig. 3 therefore show (curves with triangles) that the spin-flip excitation cross sections essentially go to zero below $10^{-3} \mathrm{~cm}^{-1}$, although appearing to be fairly substantial at collision energies larger than $10^{-2} \mathrm{~cm}^{-1}$.

The effects which are seen when selecting higher internal states of the target system could be illustrated by the calculations reported in the panels of Figs. 5 and 6, where their behavior is examined in some detail.
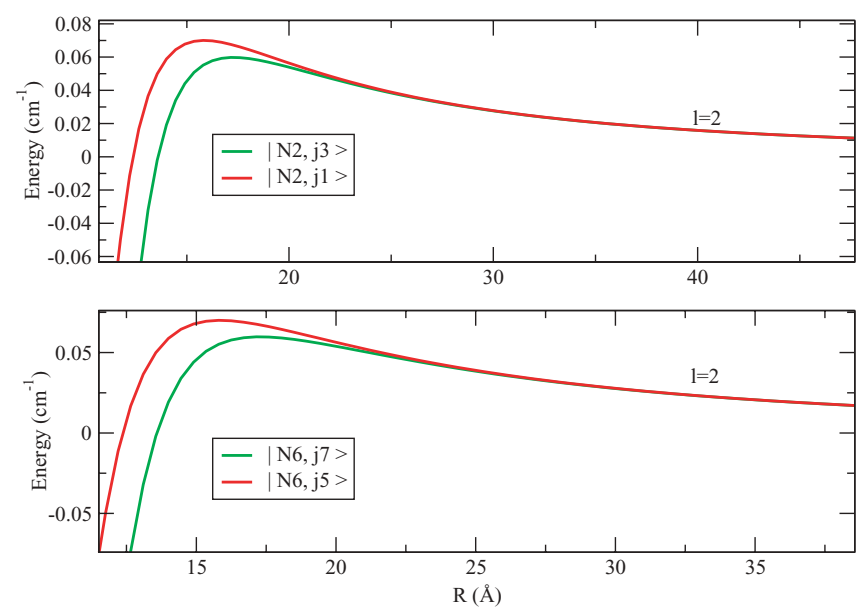

FIG. 4. (Color online) A pictorial view of the additional outgoing barriers for a few indicative states of the $\mathrm{Cs}_{2}$ target. See main text for details 


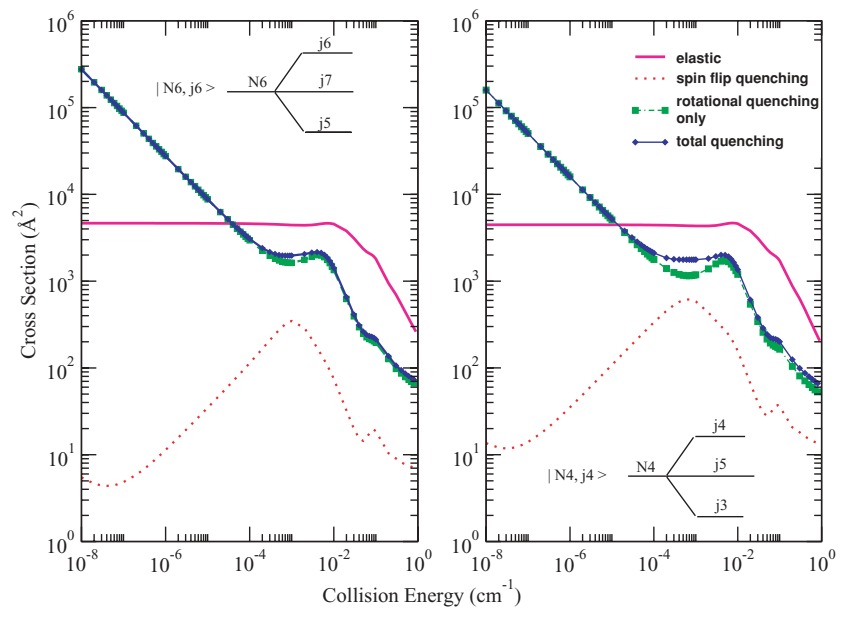

FIG. 5. (Color online) Computed elastic and quenching cross sections form the $N=6$ and $N=4$ initial levels, cosidering in both cases the top level $(j=N)$ the fine-structure state.

The calculations given in the two panels of Fig. 5 represent only internal quenching processes, since the initial levels correspond in both cases to the top sublevels of their fine-structure splittings. Hence, we see that to start with higher $N$ values naturally creates a higher density of lower levels accessible during the quenching collision and therefore causes the cumulative quenching cross sections (curves with lozenges) to start diverging at relatively higher energy with respect to those of Fig. 3. In other words, the availability of a larger number of states for the downward transitions allows the system to achieve the dominance of $s$-wave scattering contributions (Wigner's regime) at relatively higher collision energies than before. As a consequence of it, we see that internal quenching probabilities become much larger than the translational cooling (elastic) cross section from about $10^{-4}$ to $10^{-5} \mathrm{~cm}^{-1}$ energy values. Furthermore, we notice that the spin-flip quenching processes are once more affected by prevented tunneling in the outgoing channels (after finestructure transitions at low energies), followed by a resumption

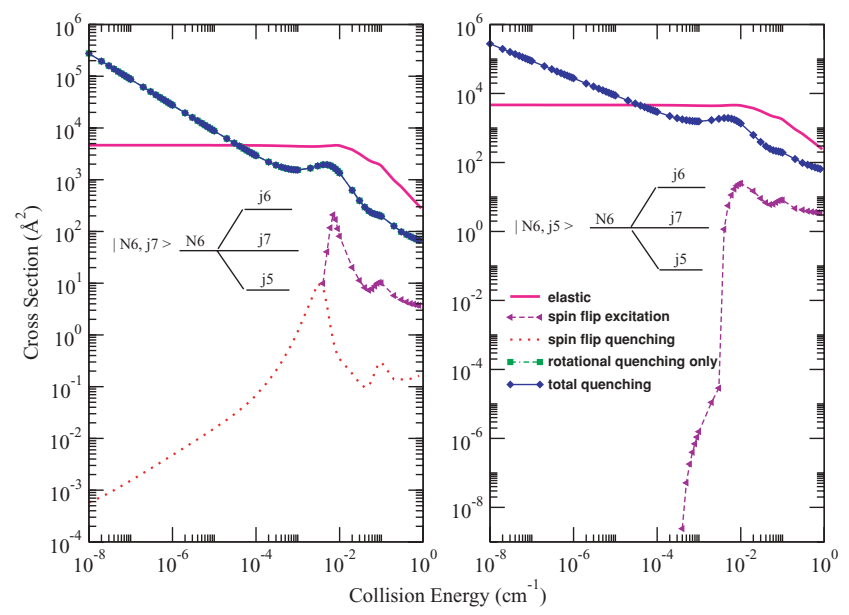

FIG. 6. (Color online) Same as in Fig. 5 but for two different initial states: $|N 6, j 7\rangle$ in the left panel and $|N 6, j 5\rangle$ in the right panel. The notation is the same as in the previous figure. of Wigner-type behavior at much lower collision energies (e.g., below $10^{-7} \mathrm{~cm}^{-1}$ ). Once again, the rotational quenching probabilities are much larger than those for the spin-flip processes and therefore dominate the cumulative (total) cross sections. The additional data of Fig. 6 are meant to remind us of a different effect, i.e., of the size of the excitation probabilities between fine-structure levels, at the relatively higher energies of $10^{-2}-10^{-3} \mathrm{~cm}^{-1}$. At those energies, and starting with initial molecular states in different fine-structure levels, we have the possibility that the available relative translational energies can open transitions between fine-structure levels. Thus, we see fairly large excitation cross sections which are larger than the computed quenching processes of spin-flip between those same levels. One should expect, therefore, that the collisional outcomes may end up producing a predominance of molecules in the highest levels of the fine-structure splittings associated with the available $N$ states of the rotating triplet molecule. As expected, however, such excitation processes become closed when the collision energy decreases and cease to contribute to trap "heating" into the higher levels of the fine-structure manifolds as the collision energy goes below $10^{-4} \mathrm{~cm}^{-1}$. Another interesting feature which can be followed when analyzing the behavior of our system is to get data on the possible importance of isotopic changes. Thus, we present in Fig. 7 the behavior of the elastic and quenching cross sections for the title molecule colliding with both ${ }^{3} \mathrm{He}$ and ${ }^{4} \mathrm{He}$ as atomic partners. The chosen initial states of $\mathrm{Cs}_{2}$ are levels associated with the highest fine-structure state: $|N 2, j 2\rangle$ and $|N 4, j 4\rangle$.

The lighter partner is clearly seen to generate markedly larger elastic cross sections in both cases examined, although both of them reach large sizes $\left(>10^{4} \AA^{2}\right)$ already at collision energies of about $10^{-3} \mathrm{~cm}^{-1}$. On the other hand, the cumulative quenching cross sections which are summed over all available final states (including spin-flipping transitions), indicate the heaver isotope of ${ }^{4} \mathrm{He}$ to produce more efficient quenching probabilities: the initial "damping" of the cross sections at the energies around $10^{-3} \mathrm{~cm}^{-1}$ is much more marked for ${ }^{3} \mathrm{He}$ projectiles than for ${ }^{4} \mathrm{He}$. One could therefore say that the quenching probabilities become larger than the cooling

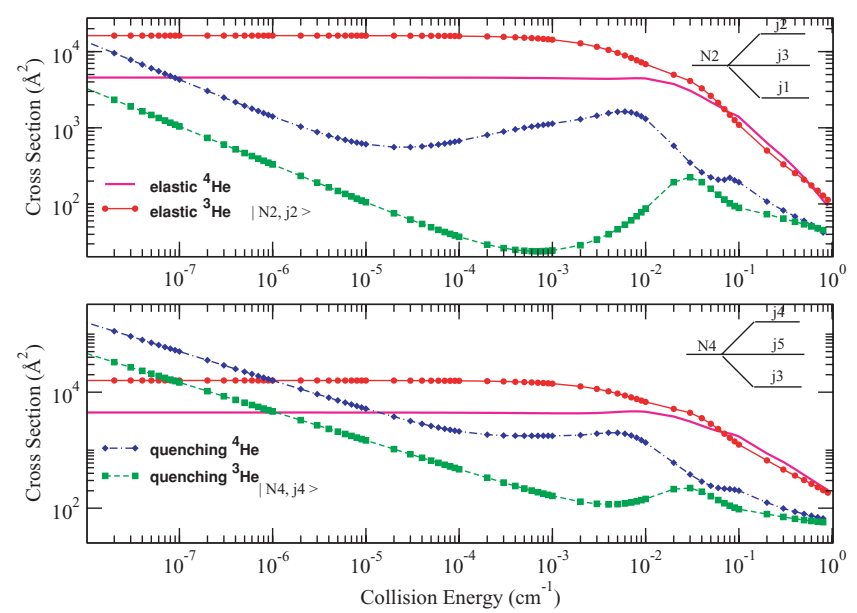

FIG. 7. (Color online) Comparison of computed scattering cross sections for ${ }^{3} \mathrm{He}$ (red dots) and ${ }^{4} \mathrm{He}$ (dotted curves) and quenching cross sections at low and ultralow collision energies. 


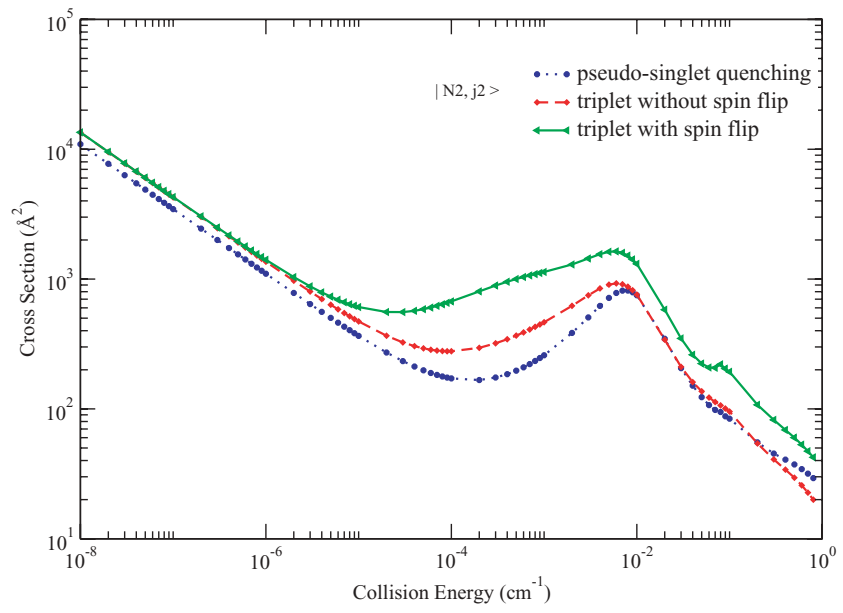

FIG. 8. (Color online) Computed cumulative inelastic cross sections using the full potential energy surface plus spin-rotation and spin-spin terms (triangles and lozenges) and cross sections using a simplified, pseudosinglet potential (full circles). See text for further details

probabilities much sooner (i.e., at higher energies) in the case of ${ }^{4} \mathrm{He}$ than of ${ }^{3} \mathrm{He}$. In the lower panel, in fact, we see the quenching efficiency of the ${ }^{4} \mathrm{He}$ gets larger than its cooling efficiency around $10^{-5} \mathrm{~cm}^{-1}$, while this change occurs only below $10^{-7} \mathrm{~cm}^{-1}$ for ${ }^{3} \mathrm{He}$ as a projectile. This is a substantial difference which is going to clearly influence the isotopic choices in the possible trap setups which one may wish to consider for the present system. Another, possible probing of the ultralow energies dynamics is provided by a sort of "computational tuning" of the interaction, whereby the target molecule is treated as a "pseudosinglet" species and therefore the interaction couplings from the spin-rotational terms discussed in the earlier sections are disregarded. To test the changes in the cumulative quenching probabilites, we report in Fig. 8 the behavior of the total inelastic cross sections for three different situations:

(a) when the $\mathrm{Cs}_{2}$ target is treated as a pseudosinglet species, thereby eliminating both spin-rotational couplings and the options of producing spin-flipping rearrangements, as discussed earlier;

(b) the correct treatment of the full dynamics with the additional presence of the spin-rotation coupling, thereby producing cumulative inelastic cross sections which include spin-flip processes; and

(c) the use of the same full interaction but extracting only inelastic contributions which do not include spin-flipping effects.

The calculations of Fig. 8 clearly show significant differences in the low-energy range of collision energies, between $10^{-2}$ and $10^{-5} \mathrm{~cm}^{-1}$. However, when one moves down to the ultralow collision energy range below $10^{-5} \mathrm{~cm}^{-1}$, one sees that all processes enter the $s$-wave dominance of the quantum dynamics [37] and that the differences essentially disappear. This means that one could use the simpler physical picture which disregards spin effects and thus obtain at ultralow energies the same cumulative inelastic cross sections: the latter are dominated by quenching transitions that largely involve

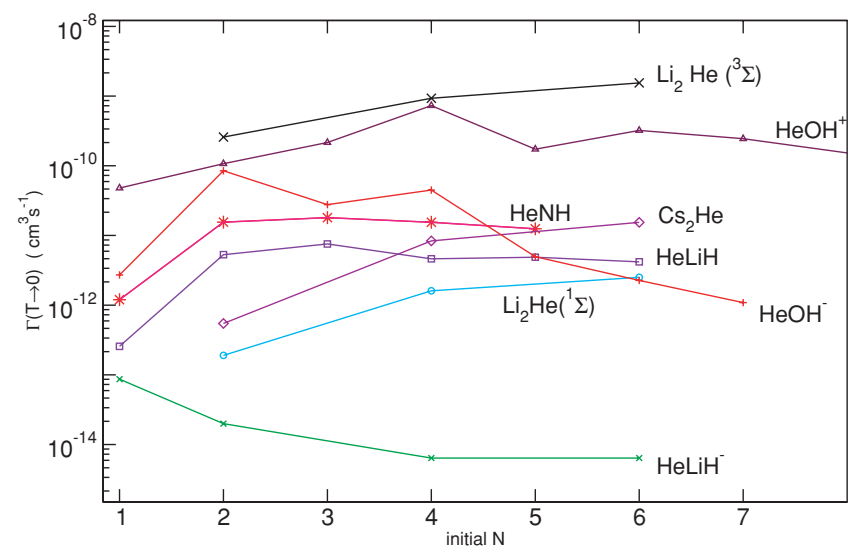

FIG. 9. (Color online) Computed cumulative deexcitation rates at vanishing temperature, as a function of the initial rotor states $|N\rangle$. See main text for details. The data shown are $\mathrm{HeOH}^{+}$[39], $\mathrm{HeNH}$ [40], $\mathrm{HeLiH}\left({ }^{1} \Sigma^{+}\right)$[41], $\mathrm{Li}_{2}\left({ }^{1} \Sigma_{g}^{+}\right) \mathrm{He}$ [42], $\mathrm{HeOH}^{-}$[43], $\mathrm{HeLiH}^{-}$[44], and $\mathrm{Li}_{2}\left({ }^{3} \Sigma^{+}\right) \mathrm{He}[45]$.

those rotational states of the target which are decoupled from electronic spin effects.

Useful common global quantities that provide us with indications on the quenching efficiency of the partner molecule are the cumulative deexcitation rates from a range of initial internal molecular states. Over the years we have generated such quantities for a broad range of molecules and therefore report a sampling of such values in comparison with those computed for the present system. The calculatons were carried out at the lowest collision energy of $10^{-9} \mathrm{~cm}^{-1}$.

A perusal through the data reported by Fig. 9 allows us to make the following considerations:

(a) The strong interaction between cationic species and $\mathrm{He}$ is clearly visible from the behavior of the $\mathrm{He}^{-\mathrm{OH}^{+}}$system, which provides one of the most efficient partners for collisional quenching by buffer He gas [39].

(b) We also see that a light (neutral molecule) rotor in a spin-stretched state, the $\operatorname{Li}_{2}\left({ }^{3} \Sigma_{u}^{+}\right)$target, provides the largest relaxation rates of the figure, indicating a very large efficiency of rotational energy quenching by collisions, as discussed in Refs. [27,45] for spinstretched dimers.

(c) By the same token, we see that anionic species that are only weakly interacting with the buffer gas $\left(\mathrm{LiH}^{-}\right.$and $\left.\mathrm{OH}^{-}\right)$ are much less efficiently quenched by collisions with the latter and therefore yield fairly small relaxation rates, even smaller than those for the present system.

(d) The present $\mathrm{Cs}_{2}-\mathrm{He}$ case involves an electronically "spin-stretched" molecular partner and also shows fairly large quenching rates. It also exhibits a rather smaller rotational constant $\left[0.0054\right.$ vs. $0.276 \mathrm{~cm}^{-1}$ for $\left.\operatorname{Li}_{2}\left({ }^{3} \Sigma_{u}^{+}\right)\right]$and is more weakly interacting with the helium buffer gas. Hence, in comparison with $\mathrm{Li}_{2}$, the collisional quenching of its internal states becomes a less efficient process than that of the latter, although remaining still fairly effective. In conclusion, in spite of the rather weak interaction between $\mathrm{He}$ and $\mathrm{Cs}_{2}\left({ }^{3} \Sigma_{u}^{+}\right)$, we have found the process of its collisional quenching to be a rather efficient mechanism at ultralow energies, as suggested by the anisotropy of its interaction and in spite of the marked weakness of the latter. 


\section{E. Present conclusions}

With the computational work described in the present article we have examined, within an accurate quantum coupledchannels method, the relative efficiencies of translational cooling and internal rotational quenching of $\mathrm{Cs}_{2}\left({ }^{3} \Sigma_{u}^{+}\right)$molecules that are interacting at ultralow collision energies with a buffer gas of either ${ }^{4} \mathrm{He}$ or ${ }^{3} \mathrm{He}$ atoms. Because of the special consequences of having a triplet spin state in the target dimer molecule, we had to generate the necessary spin-rotation and spin-spin coupling constants from a first principle calculation and then use our results to correctly describe the rotational levels of the target, plus its fine-structure level-splitting correction. We have also calculated from first principles the electronic potential energy surface of the triatomic complex, as a function of its three Jacobi coordinates $(R, r, \theta)$ and numerically generated the vibrational bound state of the $\mathrm{Cs}_{2}\left({ }^{3} \Sigma_{u}^{+}\right)$species. We have further produced a vibrationally averaged intermolecular potential for the vibrational ground state, i.e., the $V_{00}(R, \theta)$ PES, using it in turn to evaluate elastic and rotationally inelastic cross sections down to ultralow values (around $10^{-8} \mathrm{~cm}^{-1}$ ) of relative translational energy. Our aim has been to find out, for a system like $\mathrm{Cs}_{2}\left({ }^{3} \Sigma_{u}^{+}\right)$ of current experimental interest, how efficiently it could be cooled by sympathetic collision with $\mathrm{He}$ as a buffer gas and how the cooling channels compare in size with the collisional internal quenching of rotational energy or of spin inversion processes. The results of the present computation could then be summarized as follows:

- In spite of the rather weak interactions of the $\mathrm{Cs}_{2}$ in its spin-stretched state with helium, it undergoes fairly efficient rotational quenching by collisions, while preferring ${ }^{4} \mathrm{He}$ as a more efficient quenching partner with respect to ${ }^{3} \mathrm{He}$.

- The collisions leading to translational cooling make the latter channel to be preferred over internal collisional quenching down to temperatures of about $10^{-4} \mathrm{~K}$, below which the rotational quenching cross sections take over in size, especially when internally hotter $\mathrm{Cs}_{2}\left({ }^{3} \Sigma_{u}^{+}\right)$molecules are considered.

- When one compares the rotational quenching rates with those of other systems (e.g., see the data shown by Fig. 9) it is found that $\mathrm{Cs}_{2}\left({ }^{3} \Sigma_{u}^{+}\right)$yields fairly large rates with respect to other weakly interacting rotors but still smaller than other spinstretched dimers like $\mathrm{Li}_{2}$ or strongly interacting cations like $\mathrm{OH}^{+}$. Hence, we could say that the smallness of the $B$ value of $\mathrm{Cs}_{2}\left({ }^{3} \Sigma_{u}^{+}\right)$plays a significant role in enhancing rotational quenching rates together with the marked anisotropy of its PES as analyzed in Sec. II.

Since the present $\mathrm{Cs}_{2}$ dimer also supports a large number of vibrational bound states, it is also of interest to further explore those channels to get information on their quenching efficiency at ultralow temperatures; this will be the subject of a further study currently carried out in our laboratory.

\section{ACKNOWLEDGMENTS}

The financial support of the Italian Ministry of Research (MIUR) through its PRIN program is acknowledged together with the computational support of the CASPUR Consortium. The financial support from the Research Committee of the University of Rome "Sapienza" is also acknowledged.
[1] S. Haroche, Phys. Rev. Lett. 101, 160001 (2008).

[2] J. Doyle, B. Friederich, R. V. Krems, and F. Masnou-Seeuws, Eur. Phys. J. D 31, 149 (2004).

[3] W. Ketterle, Chem. Phys. Chem. 3, 736 (2002).

[4] D. De Mille, Phys. Rev. Lett. 88, 067901 (2002).

[5] D. Hershbach, Rev. Mod. Phys. 71, S411 (1999).

[6] J. M. Doyle and B. Friederich, Nature 401, 749 (1999).

[7] C. A. R. Sa De Melo, Phys. Today 61, 45 (2008).

[8] F. A. Gianturco and M. Tacconi, Faraday Discuss. 142, 463 (2009).

[9] L. Santos, G. V. Shlyapnikov, P. Zoller, and M. Lewenstein, Phys. Rev. Lett. 85, 1791 (2000).

[10] F. Masnou-Seeuws and P. Pillet, Adv. At. Mol. Phys. 47, 54 (2001).

[11] J. D. Weinstein, R. de Carvalho, T. Giullet, B. Friederich, and J. M. Doyle, Nature 395, 148 (1998).

[12] R. de Carvalho, J. M. Doyle, B. Friederich, T. Guillet, J. Kum, D. Patterson, and J. D. Weinstein, Eur. Phys. J. D 7, 289 (1999).

[13] O. Dulieu, M. Raoult, and E. Tiemann, J. Phys. B 39, 19 (2006).

[14] A. Fioretti, D. Comparat, A. Crubellier, O. Dulieu, F. MasnouSeeuws, and P. Pillet, Phys. Rev. Lett. 80, 4402 (1998).

[15] C. M. Dion, C. Drag, O. Dulieu, B. Laburthe Tolra, F. MasnouSeeuws, and P. Pillet, Phys. Rev. Lett. 86, 2253 (2001).
[16] M. J. Frisch, G. W. Trucks, H. B. Schlegel, G. E. Scuseria, M. A. Robb, J. R. Cheeseman, J. A. Montgomery Jr., T. Vreven, K. N. Kudin, J. C. Burant et al., Computer program GAUSSIAN03, revision B.05, Gaussian Inc., Wallingford CT, 2004.

[17] P. J. Hay and W. R. Wadt, J. Chem. Phys. 82, 299 (1985).

[18] T. Leininger, A. Nicklass, W. Küchle, H. Stoll, M. Dolg, and A. Bergner, Chem. Phys. Lett. 255, 274 (1996).

[19] I. S. Lim, Peter Schwerdtfeger, B. Metz, and H. Stoll, J. Chem. Phys. 122, 104103 (2005).

[20] R. B. Ross, J. M. Powers, T. Atashroo, W. C. Ermler, 1. A. LaJohn, and P. A. Christiansen, J. Chem. Phys. 93, 6654 (1999).

[21] F. Xie, V. B. Sockov, A. M. Lyyra, D. Li, S. Ingram, J. Bai, V. S. Ivanov, S. Magnier, and L. Li, J. Chem. Phys. 130, 051102 (1990).

[22] M. Foucrault, Ph. Millie, and J. P. Daudey, J. Chem. Phys. 96, 1257 (1992).

[23] D. Li, F. Xie, L. Li, S. Magnier, V. B. Sovkov, and V. S. Ivanov, Chem. Phys. Lett. 441, 39 (2007).

[24] S. F. Boys and F. Bernardi, Mol. Phys. 19, 553 (1970).

[25] O. Dulieu (private communication, 2008).

[26] R. Prosmiti, G. Delgado-Barrio, P. Villareal, E. Yurtsever, E. Coccia, and F. A. Gianturco, J. Phys. Chem. A 113, 14718 (2009). 
[27] S. Bovino, E. Bodo, E. Yurtsever, and F. A. Gianturco, J. Chem. Phys. 128, 224312 (2008).

[28] J. M. Brown, J. T. Hougen, K. T. Huber, J. W. C. Johns, I. Kopp, H. Levebvre-Brion, A. J. Meter, D. A. Ramsay, J. Rostas, and R. N. Zare, J. Mol. Spectrosc. 55, 500 (1995).

[29] G. C. Corey, M. H. Alexander, and J. Schaefer, J. Chem. Phys. 85, 2726 (1986).

[30] J. T. Hougen, Natl. Bur. Stand. (US) Monogr. 115, 109 (1970).

[31] G. C. Corey and F. R. McCourt, J. Phys. Chem. 87, 2723 (1983).

[32] R. Martinazzo, E. Bodo, and F. A. Gianturco, Comput. Phys. Commun. 151, 187 (2003).

[33] O. Vahtras, B. Minaev, and H. Agren, Chem. Phys. Lett. 281, 186 (1997).

[34] DALTON, a molecular electronic structure program, Release 2.0 (2005), see http://www.kjemi.uio.no/software/dalton/ dalton.html.

[35] N. Balakrishnan, R. C. Forrey, and A. Dalgarno, Phys. Rev. Lett. 80, 3224 (1998).
[36] N. Balakrishnan, R. C. Forrey, and A. Dalgarno, J. Chem. Phys. 113, 621 (1997).

[37] E. P. Wigner, Phys. Rev. 73, 1002 (1948).

[38] N. Balakrishnan, G. Groenenboom, R. Krems, and A. Dalgarno, J. Chem. Phys. 118, 7386 (2003).

[39] L. Gonzalez-Sanchez, E. Bodo, and F. A. Gianturco, Eur. Phys. J. D 44, 65 (2007).

[40] R. V. Krems, H. R. Sadeghpour, A. Dalgarno, D. Zgid, J. Kłos, and G. Chałasiński, Phys. Rev. A 68, 051401 (2003).

[41] E. Bodo and F. A. Gianturco, J. Phys. Chem. A 107, 7328 (2003).

[42] E. Bodo, F. A. Gianturco, F. Sebastianelli, E. Yurtsever, and M. Yurtsever, Theor. Chem. Acc. 112, 263 (2004).

[43] L. Gonzalez-Sanchez, F. Marinetti, E. Bodo, and F. A. Gianturco, J. Phys. B 39, S1203 (2006)

[44] D. Lopez-Duràn, M. Tacconi, and F. A. Gianturco, Eur. Phys. J. D 55, 601 (2009).

[45] L. Gonzalez-Sanchez, E. Bodo, and F. A. Gianturco, J. Chem. Phys. 127, 244315 (2007). 